\title{
Clinical Study \\ Status of the Gastric Mucosa with Endoscopically Diagnosed Gastrointestinal Stromal Tumor
}

\author{
Kouichi Nonaka, ${ }^{1,2}$ Shinichi Ban, ${ }^{3}$ Yoshimitsu Hiejima, ${ }^{4}$ Rei Narita, ${ }^{1}$ Michio Shimizu, ${ }^{5}$ \\ Masayasu Aikawa, ${ }^{6}$ Ken Ohata, ${ }^{2}$ Nobuyuki Matsuhashi, ${ }^{2}$ Shin Arai, ${ }^{1}$ and Hiroto Kita ${ }^{1}$ \\ ${ }^{1}$ Department of Gastroenterology, Saitama Medical University International Medical Center, 1397-1 Yamane, Hidaka, \\ Saitama 350-1298, Japan \\ ${ }^{2}$ Department of Gastroenterology, NTT Medical Center Tokyo, 5-9-22 Higashi-gotanda Shinagawa-ku, Tokyo 141-8625, Japan \\ ${ }^{3}$ Department of Pathology, Saiseikai Kawaguchi General Hospital, 5-11-5 Nishikawaguchi, Kawaguchi, Saitama 332-8558, Japan \\ ${ }^{4}$ Graduate School of Healthcare, Tokyo Healthcare University, 3-11-3 Setagaya, Setagaya-ku, Tokyo 154-8568, Japan \\ ${ }^{5}$ Department of Pathology, Saitama Medical University International Medical Center, 1397-1 Yamane, Hidaka, \\ Saitama 350-1298, Japan \\ ${ }^{6}$ Department of Surgery, Saitama Medical University International Medical Center, 1397-1 Yamane, Hidaka, Saitama 350-1298, Japan
}

Correspondence should be addressed to Kouichi Nonaka; nonaka513@gmail.com

Received 8 March 2014; Revised 19 June 2014; Accepted 19 June 2014; Published 2 July 2014

Academic Editor: Tony C. K. Tham

Copyright (c) 2014 Kouichi Nonaka et al. This is an open access article distributed under the Creative Commons Attribution License, which permits unrestricted use, distribution, and reproduction in any medium, provided the original work is properly cited.

Background. Since gastrointestinal stromal tumor (GIST) is a mesenchymal submucosal tumor, the endosonographic, CT, and MRI features of gastric GISTs have been widely investigated. However, the GIST-bearing gastric mucosa status has not been reported. Objective. To characterize the GIST-bearing gastric mucosa status in terms of the degree of inflammation and atrophy, assessed endoscopically. Subjects and Methods. The subjects were 46 patients with submucosal tumors (histologically proven gastric GISTs) who had undergone upper gastrointestinal endoscopy in our hospital between April 2007 and September 2012. They were retrospectively evaluated regarding clinicopathological features, the endoscopically determined status of the entire gastric mucosa (presence or absence and degree of atrophy), presence or absence and severity of endoscopic gastritis/atrophy (A-B classification) at the GIST site, and presence or absence of $H$. pylori infection. Results. Twenty-three patients had no mucosal atrophy, but 17 and 6 had closed- and open-type atrophy, respectively. Twenty-six, 5, 12, 1, 1, and 1 patients had grades B0, B1, B2, B3, A0, and A1 gastritis/atrophy at the lesion site, respectively, with no grade A2 gastritis/atrophy. Conclusion. The results suggest that gastric GISTs tend to arise in the stomach wall with $\mathrm{H}$. pylori-negative, nonatrophic mucosa or $\mathrm{H}$. pylori-positive, mildly atrophic mucosa.

\section{Introduction}

Gastric gastrointestinal stromal tumor (GIST) is a mesenchymal tumor arising from the muscularis propria of the gastric wall and is covered with normal mucosa, giving the endoscopic appearance of a submucosal tumor with bridging folds. Since gastric GIST is a submucosal tumor, its endosonographic, CT, and MRI features have been widely investigated [1-4]. However, the status of the GIST-bearing gastric mucosa itself has not been reported. Based on our experience, many GIST patients often show no evidence of $H$. pylori infection, gastritis, or mucosal atrophy on upper gastrointestinal endoscopy, and even $H$.pylori-infected patients may show mild gastritis in the background gastric mucosa. We consider that it is necessary to evaluate the GISTbearing gastric mucosa using available data before testing the validity of this empirical rule and investigate the reason for it, if it is valid.

In this study, we retrospectively evaluated patients with submucosal tumors (histologically proven gastric GISTs) regarding the age, gender, site and size of the lesion, risk classification of GISTs, presence or absence and degree of endoscopic atrophy in the entire gastric mucosa, presence or absence and severity of endoscopic gastritis and mucosal 


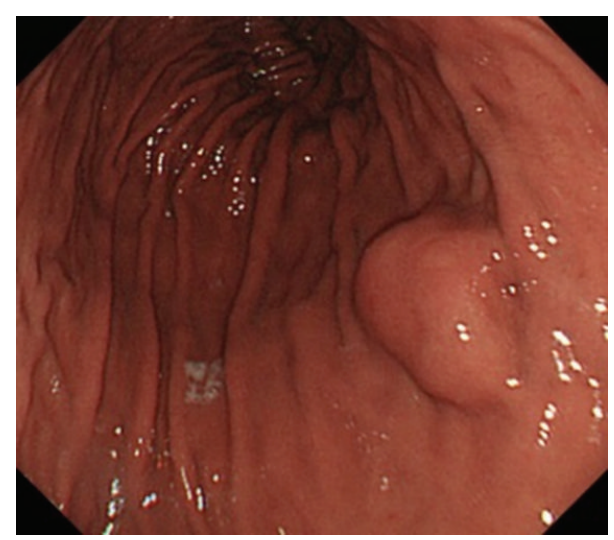

FIGURE 1: Endoscopic appearance of gastric mucosa of $H$. pyloriand atrophy-negative patient. Conventional endoscopic appearance. A $20 \mathrm{~mm}$ submucosal tumor was observed in the posterior wall of the middle gastric body. Conventional endoscopic findings were considered negative for H. Pylori.

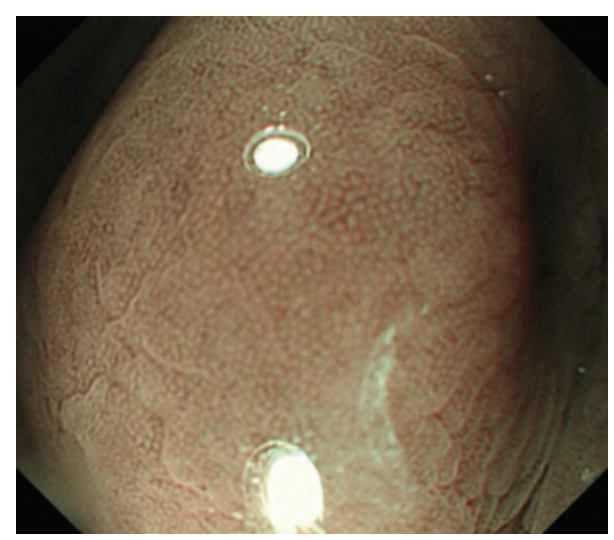

Figure 2: NBI-magnifying endoscopic findings. The lesion was classified as B0 according to Yagi's A-B classification system.

atrophy at the lesion site, and presence or absence of $H$. pylori infection.

\section{Subjects and Methods}

The subjects were 46 patients with submucosal tumors (histologically proven gastric GISTs) who had undergone both conventional (Figure 1) and NBI endoscopy (Figure 2) of the upper gastrointestinal tract in our hospital between April 2007 and September 2012. All subjects were derived from our hospital pathology database.

All of them had undergone endoscopic ultrasonography: EUS (Figure 3). Of these patients, 40 had undergone surgical resection of the tumor, but 3 had not.

These 46 patients were retrospectively evaluated regarding the age, gender, site and size of the lesion, risk classification of GISTs, presence or absence and degree of endoscopic atrophy in the entire gastric mucosa, presence or absence and severity of endoscopic gastritis and atrophy at the lesion site, and presence or absence of $H$. pylori infection. The patients

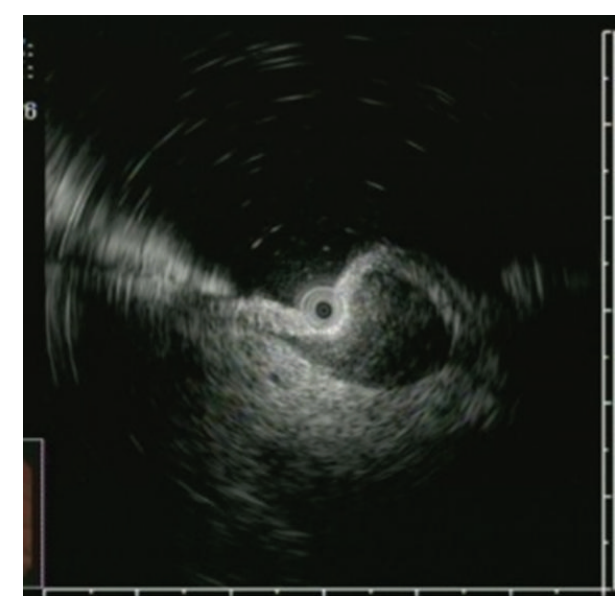

FIgURE 3: EUS findings. A homogeneous, hypoechoic tumor continuous with the fourth layer was observed.

whose tumors were resected were evaluated regarding the maximum tumor diameter of the resected specimen, and those who did not undergo tumorectomy were assessed regarding the maximum tumor diameter determined by EUS. The patients were evaluated regarding the lesion site and risk classification of GISTs according to the 14th edition of the Japanese Classification of Gastric Carcinoma [5].

Endoscopic findings were evaluated by two experienced endoscopists (Kouichi Nonaka and Shinichi Ban). The presence or absence and grade of atrophy of the entire stomach were determined by conventional endoscopy and classified into nonatrophy and closed- and open-type atrophy according to the Kimura-Takemoto classification [6]. The presence or absence and severity of gastritis/atrophy were evaluated using Yagi's A-B classification system [7] based on the microsurface structure and microvascular architecture of the mucosa (Table 1). Yagi et al. defined types B0-B3 as magnified endoscopic images of the nonatrophic gastric mucosa, types A1-A2 as those of the atrophic mucosa, and type A- 0 as that of the gastric antrum in $H$. pylori-noninfected patients. For endoscopists with the skills to perform conventional upper gastrointestinal endoscopic examination and diagnosis, it is straightforward to diagnose GISTs using these two classifications.

For the diagnosis of $H$. pylori infection, all patients had undergone at least one of the following tests: histological examination of endoscopic biopsy or surgical specimens, titration of serum anti-Hp IgG antibodies, and the urea breath test. The presence of at least one positive test was considered to indicate $H$. pylori infection.

Descriptive statistics were calculated for each variable.

\section{Results}

The mean age of the 46 patients was 63.9 years (range, $45-92$ years). The male-to-female ratio was 0.92 (22 males and 24 females). Lesions were found in the upper, middle, and lower portions of the stomach of 32,12 , and 2 patients, respectively. The mean lesion diameter was $40.4 \mathrm{~mm}$ (range, 6-110 $\mathrm{mm}$ ). 
TABLE 1: Extended classification (A-B classification) of gastritis.

Magnified endoscopic image of nonatrophic mucosa

B-0: uniform round pits (crypt openings: Cos) surrounded by a regular honeycomb subepithelial capillary network (SECN) and a regular arrangement of collecting venules seen as starfish-like structures (basic appearance of an $H$. pylori-negative patient).

B-1: round pits (COs) surrounded by a regular or mild irregular honeycomb SECN; no collecting venules.

B-2: round pits (COs) and dividing sulci; no normal SECN or collecting venules.

B-3: dilated pits (COs) with more dense sulci; no normal SECN or collecting venules.

Magnified endoscopic image of atrophic mucosa or gastric antrum

A-1: ridged surface structures surrounding dilated, coiled subepithelial capillaries (SECs).

A-2: villous to granular surface structures including irregular, narrowed, coiled SECs.

Magnified image of $H$. pylori-noninfected gastric antrum

A-0: a regular tubular mucosal pattern, along which a capillary network is seen. In some cases, arcuate white zones are surrounded by round or oval white zones.

TABLE 2: Patient characteristics $(n=46)$.

\begin{tabular}{lc}
\hline Age & Mean: 63.9 years (45-92) \\
Sex $(\mathrm{M}: \mathrm{F})$ & $22: 24$ \\
Lesion site $(\mathrm{U}: \mathrm{M}: \mathrm{L})$ & $32: 12: 2$ \\
Lesion size & Mean: $40.4 \mathrm{~mm}$ \\
& $(6-110 \mathrm{~mm})$ \\
GIST risk classification & 8 \\
$\quad$ Very low risk & 25 \\
$\quad$ Low risk & 7 \\
$\quad$ Intermediate risk & 3 \\
$\quad$ High risk & \\
Status of atrophy of entire mucosa & 23 \\
$\quad$ Atrophy (一) & 17 \\
Closed-type atrophy & 6 \\
Open-type atrophy & \\
A-B classification of gastritis/atrophy & \\
at lesion site & $26: 5: 12: 1: 1: 1: 0$ \\
$\quad$ B0 : B1: B2 : B3: A0 : A1: A2 & \\
H. pylori infection & $23: 23$ \\
Positive : negative &
\end{tabular}

GISTs were classified as very low, low, intermediate, and high risk in $8,25,7$, and 3 patients, respectively. None of the patients had coexisting gastric adenoma or carcinoma.

Twenty-three patients had no mucosal atrophy, but 17 and 6 had closed- and open-type atrophy of the entire gastric mucosa, respectively. Twenty-six, 5, 12,1,1, and 1 patients had grades $\mathrm{B} 0, \mathrm{~B} 1, \mathrm{~B} 2, \mathrm{~B} 3, \mathrm{~A} 0$, and $\mathrm{A} 1$ gastritis and atrophy at the lesion site, respectively, and no patient had grade $\mathrm{A} 2$ gastritis and atrophy (Table 2).

In this study, 23 patients (50\%) had evidence of $H$. pylori infection, and the gastric mucosa of 17 and 6 of them showed closed- and open-type atrophy, respectively (Table 3). However, 4, 5, 12, 1, 0, 1, and 0 patients had grade B0, B1, B2, B3, A0, A1, and A0 gastritis/atrophy at the GIST site, respectively, and the grade $\mathrm{Al}$ gastritis/atrophy in the single patient was located in the gastric antrum. Thus, none of the patients had a clear pattern of mucosal atrophy. On the other hand, the 23 patients (50\%) with no evidence of $H$. pylori infection coincided with the 23 patients whose entire gastric mucosa showed no evidence of atrophy (atrophy [0]), and the mucosa at the GIST site showed little or no inflammation/atrophy, as indicated by the findings of: B0 (22 patients), B1 (0), B2 (0), B3 (0), A0 (1), A1 (0), and A2 (0) (Table 3).

With advancing age, the rate of $H$. pylori infection increased, and the grade of mucosal atrophy tended to become higher (Table 4). Fifty-three percent (23/43) of the patients 50 years of age or older showed evidence of $H$. pylori infection.

\section{Discussion}

This study showed that most gastric GISTs arose in the upper and middle portions of or stomach, as in previous reports [8].

Since this study involved a small number of patients, further studies involving more patients are needed. However, in this study, 3 high-risk and 8 very low-risk patients based on the GIST risk classification system had H. pylorinegative, nonatrophic gastric mucosa, indicating no correlation between the presence or absence of mucosal atrophy and grade of GIST risk.

Twenty-three (50\%) of the GIST patients and 53\% (23/43) of those 50 years of age or older were found to be positive for $H$. pylori infection. In Japan, Shiota et al. reported that the $H$. pylori infection rate in patients 50 years of age or older for the period of 2002-2006 was 60\% [9]. In 1992, Asaka et al. reported that the $H$. pylori infection rate in patients 40 years of age or older was $70 \%$ [10]; therefore, the $H$. pylori infection rate in patients 50 years of age or older in 2002, as estimated from these two studies, was about $60-70 \%$.

Assuming that the $H$. pylori infection rate was about $60-70 \%$, that rate of the GIST patients in this study was lower. Since some patients in this study were unable to undergo more than one test (e.g., tissue biopsy examination), they may have been false-negative for $H$. pylori infection. Although further studies involving more patients are needed, all of the 23 patients who were deemed $H$. pylori-positive had closedor open-type atrophic gastritis on endoscopy, and all of the 23 
TABLE 3: A-B classification of atrophy of the entire mucosa and status of the mucosa at the GIST site in H. pylori-positive and -negative patients.

\begin{tabular}{|c|c|c|c|c|c|c|c|}
\hline & B-0 & B-1 & B-2 & B-3 & A0 & $\mathrm{A} 1$ & $\mathrm{~A} 2$ \\
\hline \multicolumn{8}{|c|}{ H. pylori-negative $(n=23)$} \\
\hline Atrophy (-) & 22 & & & & 1 & & \\
\hline \multicolumn{8}{|l|}{ C-type } \\
\hline \multicolumn{8}{|l|}{ O-type } \\
\hline \multicolumn{8}{|c|}{ H. pylori-positive $(n=23)$} \\
\hline \multicolumn{8}{|l|}{ Atrophy $(-)$} \\
\hline C-type & 4 & 4 & 8 & & & 1 & \\
\hline O-type & & 1 & 4 & 1 & & & \\
\hline
\end{tabular}

C-type: closed-type atrophy, O-type: open-type atrophy.

TABLE 4: Rate of $H$. pylori infection and type of gastric mucosal atrophy by age group.

\begin{tabular}{|c|c|c|c|c|c|c|}
\hline Age group & $40-49$ & $50-59$ & $60-69$ & $70-79$ & $80-89$ & $\geq 90$ \\
\hline \multirow{2}{*}{ H. pylori-positive (\%) } & $0 / 3$ & $5 / 12$ & $11 / 21$ & $7 / 9$ & - & $0 / 1$ \\
\hline & $(0)$ & $(41.7)$ & $(52.4)$ & $(77.8)$ & - & $(0)$ \\
\hline Atrophy (-)/C-type/O-type & $3 / 0 / 0$ & $7 / 3 / 2$ & $10 / 9 / 2$ & $2 / 5 / 2$ & - & $1 / 0 / 0$ \\
\hline
\end{tabular}

C-type: closed-type atrophy, O-type: open-type atrophy.

patients who were deemed $H$. pylori-negative had $H$. pylorinoninfected gastric mucosa with no atrophy on endoscopy. Therefore, we suggest that no patients in this study were falsepositive or false-negative for $H$. pylori infection.

The following analysis is based on the assumption, from the results of the above two studies, that the $H$. pylori infection rate in patients 50 years of age or older in Japan is about $60-70 \%[9,10]$. In a hypothetical case-cohort study, in which 43 GIST patients ( $\geq 50$ years) in this study are compared to a cohort of patients ( $\geq 50$ years) in Japan, the exposure-odds ratio is 1.3-2.0, suggesting that the incidence of GIST in $H$. pylori-negative patients will be 1.3-2.0 times as high as that in their $H$. pylori-positive counterparts.

In the 23 patients who had no evidence of $H$. pylori infection, the entire gastric mucosa showed little or no atrophy on endoscopy, and the mucosa at the GIST site exhibited little or no inflammation/atrophy, as indicated by the findings based on Yagi's A-B classification system. In the 23 patients with evidence of $H$. pylori infection, the gastric mucosa showed closed- or open-type atrophy, but the gastric mucosa at the GIST site showed grade B0 gastritis in the fundic glands of 4 patients, grade B1-B3 gastritis in 18 patients, no grade A atrophy, and a grade A1 lesion in the antral pyloric glands of 1 patient. In the $4 \mathrm{H}$. pylori-positive patients with GIST in the fundic glands, the gastric mucosa at the GIST site was normal or mildly inflamed. Thus, in the patients studied, the gastric mucosa at the GIST site showed little or no atrophy.

These results suggest that gastric GIST arises from the muscularis propria underlying the $H$. pylori-negative, nonatrophic or $H$. pylori-positive, mildly atrophic gastric mucosa. None of the 46 patients had coexisting gastric adenoma or carcinoma. Although the incidence of gastric carcinomas and adenomas arising from $H$. pylori-negative, nonatrophic gastric mucosa without intestinal metaplasia is low, GISTs and other tumors may be present. Therefore, endoscopists should be prepared to perform endoscopy to avoid overlooking elevated lesions suggestive of submucosal tumors. Since this study involved a small number of patients in a single center, further studies involving more patients are needed to confirm our results.

\section{Conflict of Interests}

The authors declare that there is no conflict of interests regarding the publication of this paper.

\section{References}

[1] J. Yoshino, K. Inui, T. Wakabayashi et al., "Diagnosis and assessment of malignancy for digestive GIST-endoscopic ultrasonography and EUS-guided fine needle aspiration," Stomach and Intestine, vol. 43, pp. 163-171, 2008.

[2] S. Ohashi, S. Okamura, F. Urano, M. Maeda, H. Goto, and Y. Niwa, "Clinical malignancy risk of gist assessed by endoscopic ultrasonography," Digestive Endoscopy, vol. 18, no. 4, pp. 256262, 2006.

[3] Tateishi U. Multi-Modality Allows Diagnosis of GIST, Stomach and Intestine, vol. 43, Igaku-Shoin Ltd., Tokyo, Japan, 2008, (in Japanese with English abstract).

[4] Y. R. Choi, S. H. Kim, S. A. Kim et al., "Differentiation of Large $(\geq 5 \mathrm{~cm})$ gastrointestinal stromal tumors from benign subepithelial tumors in the stomach: radiologist performance using CT,' European Journal of Radiology, vol. 83, pp. 250-260, 2014.

[5] Japanese Research Society for Gastric Cancer, Japanese Classification of Gastric Carcinoma, Kanehara \& Company, Tokyo, Japan, 2010.

[6] K. Kimura and T. Takemoto, "An endoscopic recognition of the atrophic border and its significance in chronic gastritis," Endoscopy, vol. 3, article 87, 1969. 
[7] K. Yagi, J. Watanabe, A. Nakamura, and A. Sekine, "Magnifying views of Gastritis-AB classification," Stomach and Intestine, vol. 42, pp. 697-704, 2007 (Japanese).

[8] Y. Fujimoto, Y. Nakanishi, K. Yoshimura, and T. Shimoda, "Clinicopathologic study of primary malignant gastrointestinal stromal tumor of the stomach, with special reference to prognostic factors: analysis of results in 140 surgically resected patients," Gastric Cancer, vol. 6, no. 1, pp. 39-48, 2003.

[9] S. Shiota, K. Murakami, T. Fujioka, and Y. Yamaoka, "Population-based strategies for Helicobacter pylori-associated disease management: a Japanese perspective," Expert Review of Gastroenterology and Hepatology, vol. 4, no. 2, pp. 149-156, 2010.

[10] M. Asaka, T. Kimura, M. Kudo et al., "Relationship of Helicobacter pylori to serum pepsinogens in an asymptomatic Japanese population," Gastroenterology, vol. 102, no. 3, pp. 760-766, 1992. 


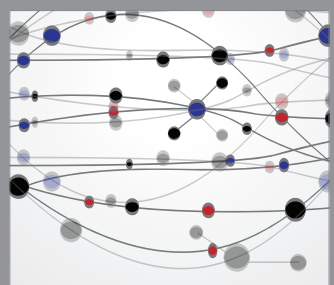

The Scientific World Journal
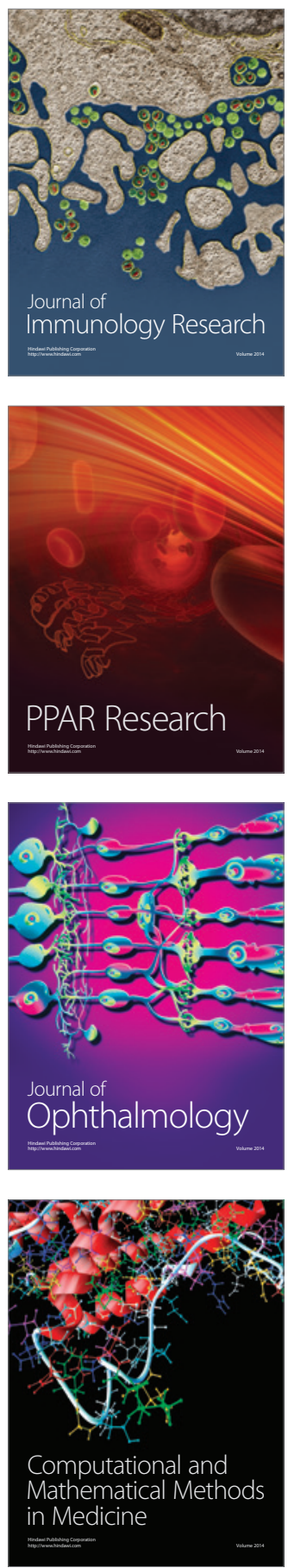

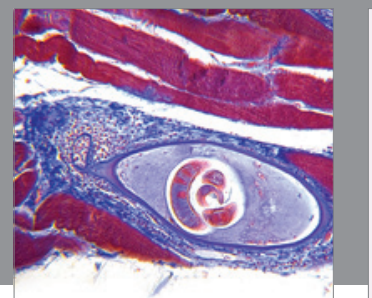

Gastroenterology

Research and Practice
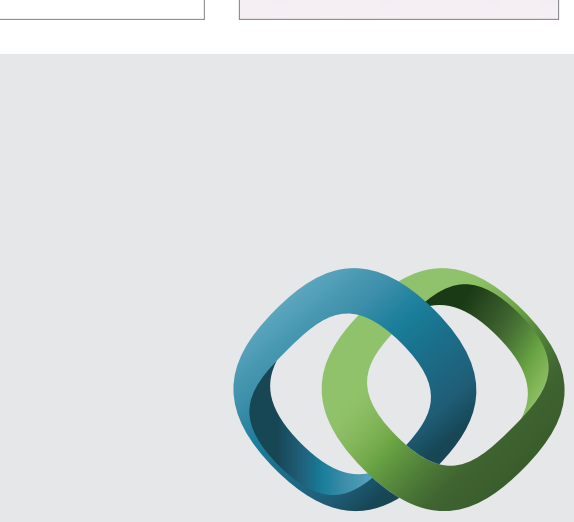

\section{Hindawi}

Submit your manuscripts at

http://www.hindawi.com
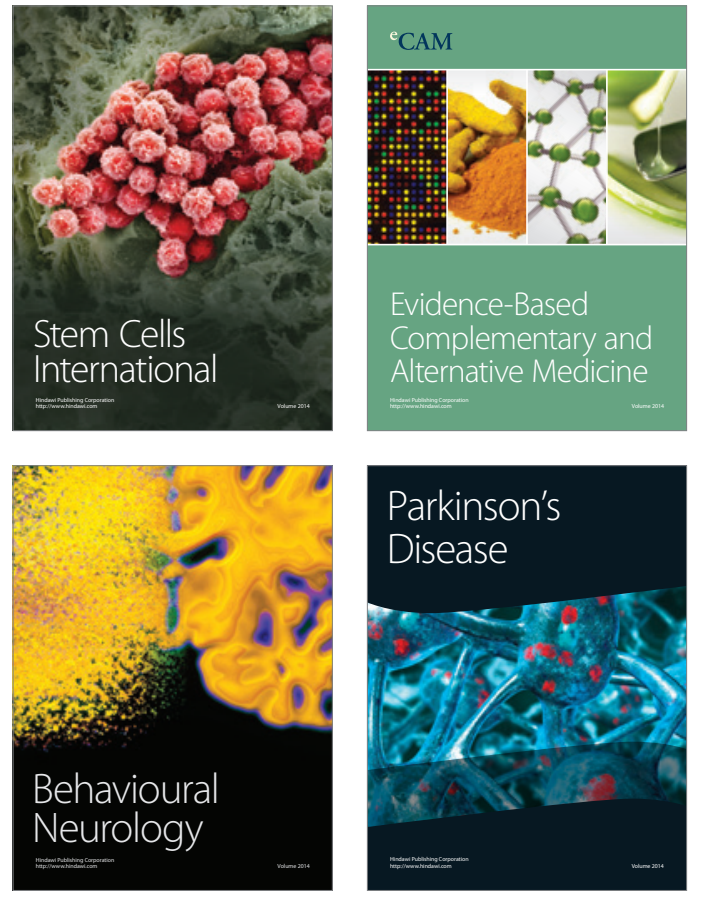
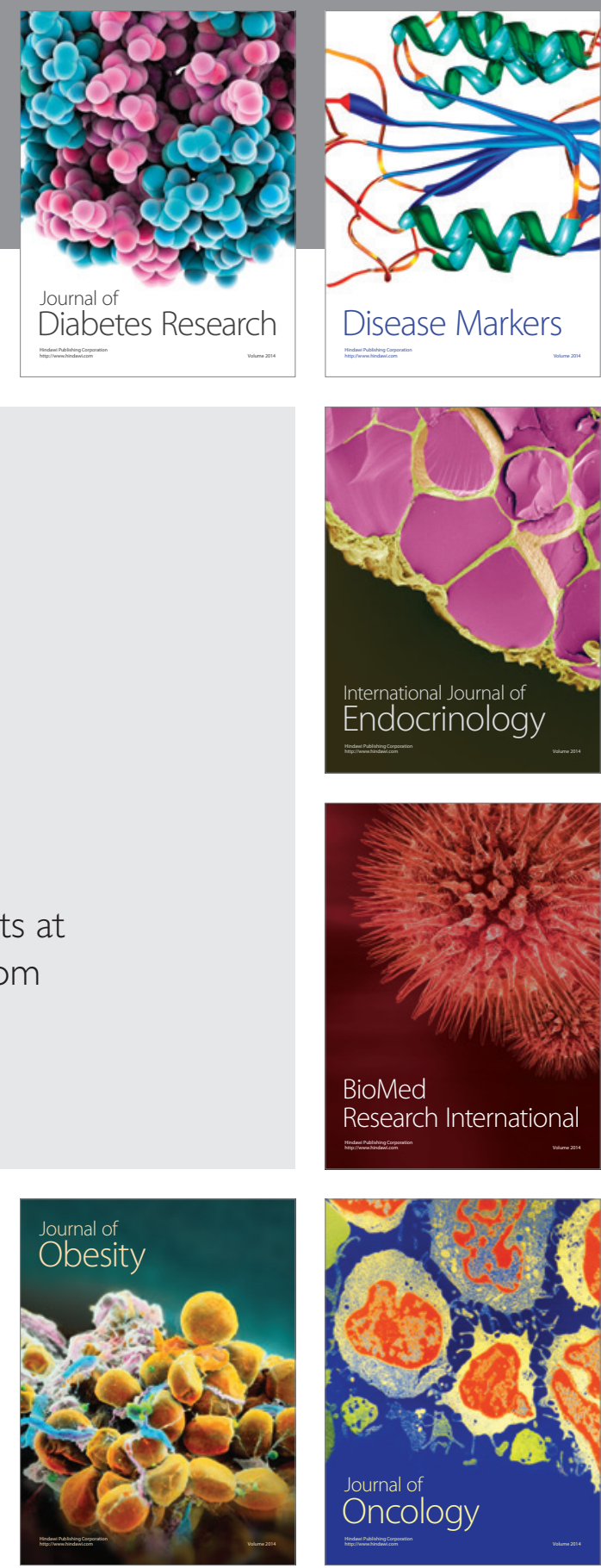

Disease Markers
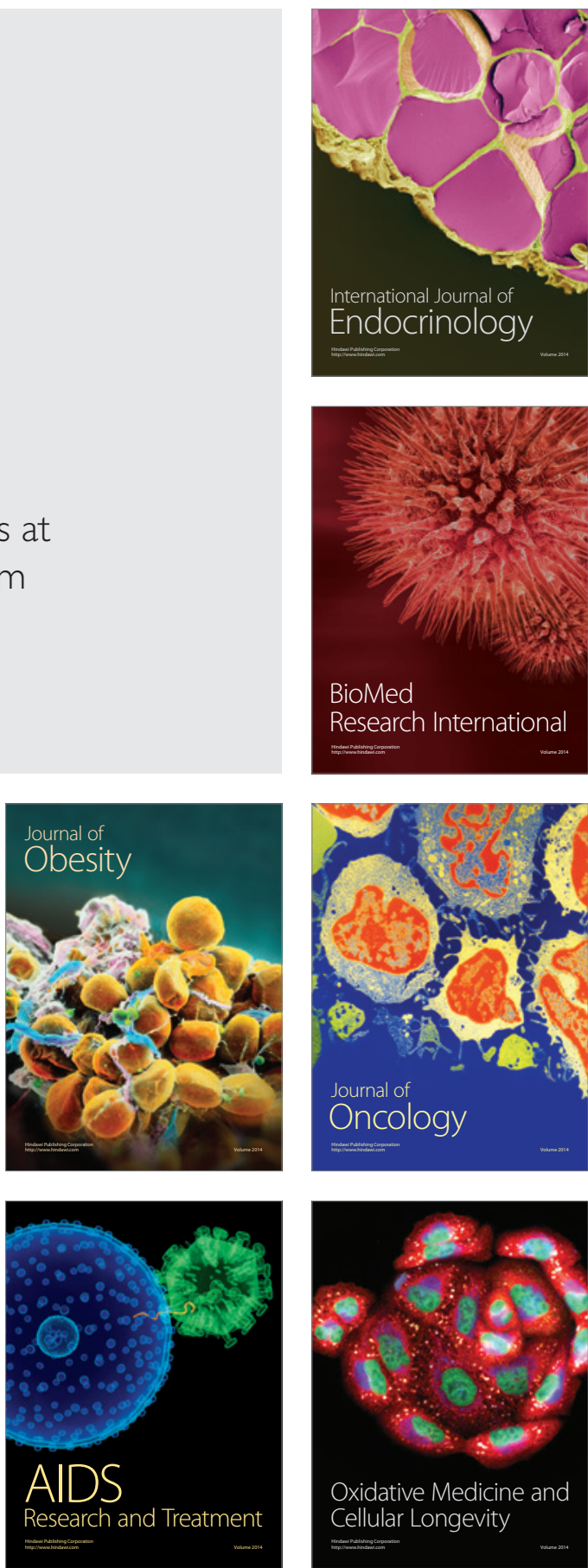